\title{
Irgarol inhibits the synthesis of ATP in mitochondria from rat liver
}

\author{
Marcantonio Bragadin ${ }^{\mathrm{a}, *}$, Francesca Cima ${ }^{\mathrm{b}}$, Loriano Ballarin ${ }^{\mathrm{b}}$, Sabrina Manente ${ }^{\mathrm{a}}$ \\ a Dipartimento di Scienze Ambientali, Università di Venezia, DD 2137, 30123 Venice, Italy \\ ${ }^{\mathrm{b}}$ Dipartimento di Biologia, Università di Padova, Via U. Bassi 58/b, 30121 Padua, Italy
}

Received 21 February 2006; received in revised form 20 July 2006; accepted 2 August 2006

Available online 18 September 2006

\begin{abstract}
The interactions of Irgarol with rat liver mithocondrial have been investigated. The results indicate that Irgarol inhibits the ATP synthesis. The analysis of the various steps involved in the ATP synthesis suggests that the inhibition is due to the opening of small-size pores.
\end{abstract}

(C) 2006 Elsevier Ltd. All rights reserved.

Keywords: Mithocondria; Irgarol; Toxicity; Pollution

\section{Introduction}

Traditionally the paint mixtures used to protect boats are deliberately designed so that they leak toxic compounds such a copper pyrithione, tributyltin, Sea-Nine and Irgarol into the sea.

These compounds are effective in preventing the growth of marine organisms (Dahl and Blank, 1996; Evans et al., 2000; Bragadin et al., 2005), but unfortunately despite the plant specificity, they also effect other non-target organisms.

Two examples of this are the Juvenile Rainbow Trout, Oncorhynchus mykiss, and the Artemia salina and the toxicity parameters are given in (Okamura et al., 2002; Panaguola et al., 2002).

As in all cases where a toxicity in whole organisms has been verified, this stimulated the researchers to individuate the molecular targets responsible for the toxicity in the whole organisms.

In many cases, the preferential target for the toxic compounds are the mitochondria: since the mitochondria are the energy source for the cell, a damage to the mitochondria gives rise to an equivalent cell damage. Since the

\footnotetext{
* Corresponding author. Tel.: +39 041234 8507; fax: +39 0412348953.

E-mail address: bragadin@unive.it (M. Bragadin).
}

mitochondria are similar in all living species, in this paper we have investigated the fact that Irgarol induces an inhibition of the ATP synthesis in mitochondria from rat liver.

All of the steps involved in the ATP synthesis have been investigated and we concluded that the inhibition of the ATP synthesis is due to the opening of a low-size membrane pore, since this phenomenon is induced by the lowest Irgarol dose.

\section{Materials and methods}

The mitochondria were prepared from the livers of fasted albino Wistar rats, weighing about $300 \mathrm{~g}$ (Bragadin et al., 1980). The mitochondrial protein concentration was determined using the Lowry et al. procedure (Lowry et al., 1951).

The ATP synthesis/hydrolysis experiments were performed in a low buffered medium, using a $\mathrm{pH}$ electrode connected to a PHM Radiometer (pH-meter) 84 (Radiometer, Copenhagen, Denmark) in order to monitor the $\mathrm{pH}$ changes which accompany the reaction: ATP $\leftrightarrow$ ADP + $\mathrm{P}_{\mathrm{i}}+\mathrm{H}^{+}$, using room temperature stirring conditions $\left(\mathrm{ATP}=\right.$ adenosine $5^{\prime}$ triphosphate, $\mathrm{ADP}=$ adenosine $5^{\prime}$ diphosphate, $\mathrm{P}_{\mathrm{i}}=$ inorganic phosphate).

The mitochondrial oxygen consumption was measured using a Clark oxygen electrode (Yellow Springs 
Instrument, Yellow Spring, OH, USA) fitted in a thermostat-controlled, closed chamber, equipped with a magnetic stirrer. The reaction medium $(2 \mathrm{ml})$ was kept thermostated at $20{ }^{\circ} \mathrm{C}$ throughout the experiments.

Following the absorbance decrease, the swelling experiments were performed at $540 \mathrm{~nm}$, in a Jenway 6400 (Felsted, Esse, UK) spectrophotometer, equipped with a stirring apparatus.

After the addition of the mitochondria to the resuspending medium, $(2.5 \mathrm{ml})$, the instrument was adjusted to zero absorbance.

All of the reagents were analytical grade. The tetramethyl-p-phenylendiamine (TMPD) reagents, the carbonylcyanide- $p$-trifluoromethoxyphenyl hydrazone (FCCP), the 2,4-dinitrophenol (DNP), the sodium glutamate, the sodium malate, the Oligomycin and the Cyclosporine, were all obtained from Sigma (Milan, Italy).

\section{Results and discussion}

As regards mitochondria the substrates arising from the Krebs cycle are oxidised by means of molecular oxygen. The oxidation occurs by means of a sequence of redox couples (the cytochromes) in the mitochondrial respiratory chain (RC). The ATP synthesis occurs as a consequence of the electron flow in the RC.

The chemiosmotic hypothesis (Mitchell, 1979) explains the mechanism which correlates the electron flow in the RC with the ATP synthesis. The theory asserts that the electron flow in the $\mathrm{RC}$ gives rise to a proton extrusion and that, since the mitochondrial (inner) membrane is not permeant to the protons, the proton extrusion gives rise to a $\Delta \mathrm{pH}$ and to a $\Delta \psi$, the proton motive force (p.m.f.)

p.m.f. $=\Delta \mathrm{pH}+\Delta \psi$

The energy which is stored in the $\mathrm{RC}$, in the form of a p.m.f., during the electron flow, is subsequently utilised to synthetize the ATP from the ADP.

Taking into account the chemiosmotic mechanism, if a toxic compound inhibits the ATP synthesis, this effect can be due to

- inhibition of the RC;

- inhibition of the ATPase (the enzyme which catalyses the ATP synthesis);

- enhancement of the proton permeability.

On its turn, the last step, the enhancement of the proton permeability, can be due to

uncoupling effect;

detergent effect;

opening of a membrane pore.

In the case of Irgarol, since this compound inhibits the ATP synthesis, as shown in Fig. 1, each step involved in the ATP synthesis has been selectively analysed, in order to find the step which requires the lowest dose of Irgarol.
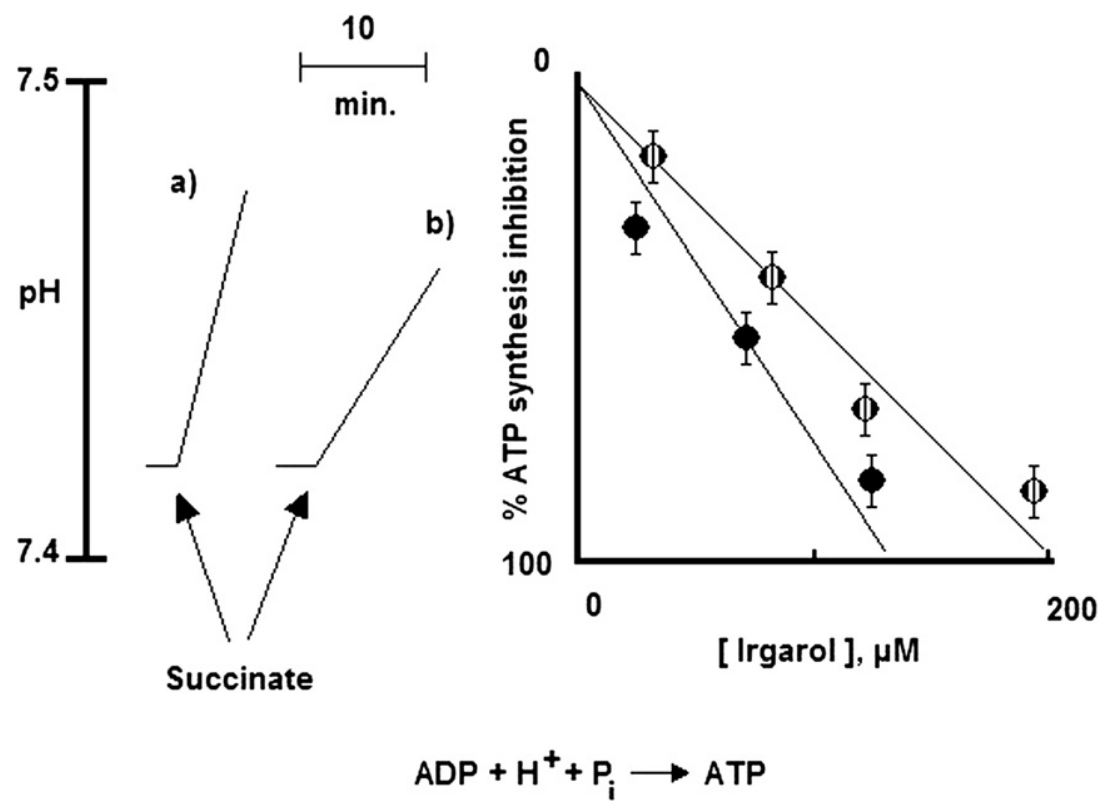

Fig. 1. ATP synthesis in mitochondria and its inhibition by Irgarol. The rate of ATP synthesis was measured using a pHmeter, following the pH changes which accompany the ADP $+\mathrm{P}_{\mathrm{i}} \rightarrow \mathrm{ATP}+\mathrm{OH}^{-}$reaction. The figure shows a typical experiment in which the rate of alkalinization is reported (a) in the absence and (b) in the presence of Irgarol. The graph reports the initial alkalinization rates in the absence $(\bullet)$ and in the presence $(O)$ of Cyclosporine, which is an inhibitor of the opening of large and small size pores. Medium composition: $0.25 \mathrm{M}$ sucrose, $0.1 \mathrm{mM}$ Hepes- $\mathrm{Mops}_{\mathrm{pH}} 7.4,2 \mathrm{mM} \mathrm{MgCl}_{2}$, $0.5 \mathrm{mM}$ EGTA, $0.5 \mathrm{mM}$ phosphate $\left(\mathrm{P}_{\mathrm{i}}\right), 0.5 \mathrm{mM}$ ADP, $2 \mathrm{mM}$ succinate. The final concentration of mitochondria was $0.5 \mathrm{mg} \mathrm{ml}^{-1}$. The succinate was added $3 \mathrm{~min}$ after the Irgarol and the Irgarol was added immediately after the mitochondria. When present, the concentratrion of cyclosporine was $1 \gamma$. Each experiment is the mean value of four experiments. 
As consequence, this step will be responsible for the toxic effect (inhibition of the ATP synthesis) of the compound.

The first step to be analysed was the RC.

\subsection{The $R C$}

The inhibition of the RC obviously implies an equivalent inhibition of the ATP synthesis. Fig. 2 shows the respiratory rate of the mitochondria (the rate of oxygen consumption) using a succinate as a substrate.

The rate is stimulated up to a maximum value by means of an uncoupler (see below).

In this condition, the addition of Irgarol gives rise to an inhibition of the respiratory rate.

The percentage of inhibition is contained in the graph in Fig. 2.
Since the electron flow in the $\mathrm{RC}$ can be induced by means of three kinds of reducing substrates, depending on their redox potential, the respiratory rate was also examined in the presence of Glutamate/Malate and ascorbic acid.

In the case of the Glutamate/Malate as a reducing substrate, the whole RC is utilised (Fig. 3), but the addition of Irgarol does not induce any inhibition in the RC (not shown).

Analogously, using ascorbic acid a substrate (in the presence of TMPD), only the third site of the RC is examined.

Once again in this case, no inhibition by the Irgarol occurs (not shown).

Taking into account the structure of the RC (Fig. 3) and the experimental results, our interpretation is that Irgarol interacts with the succinic dehydrogenase in the RC.
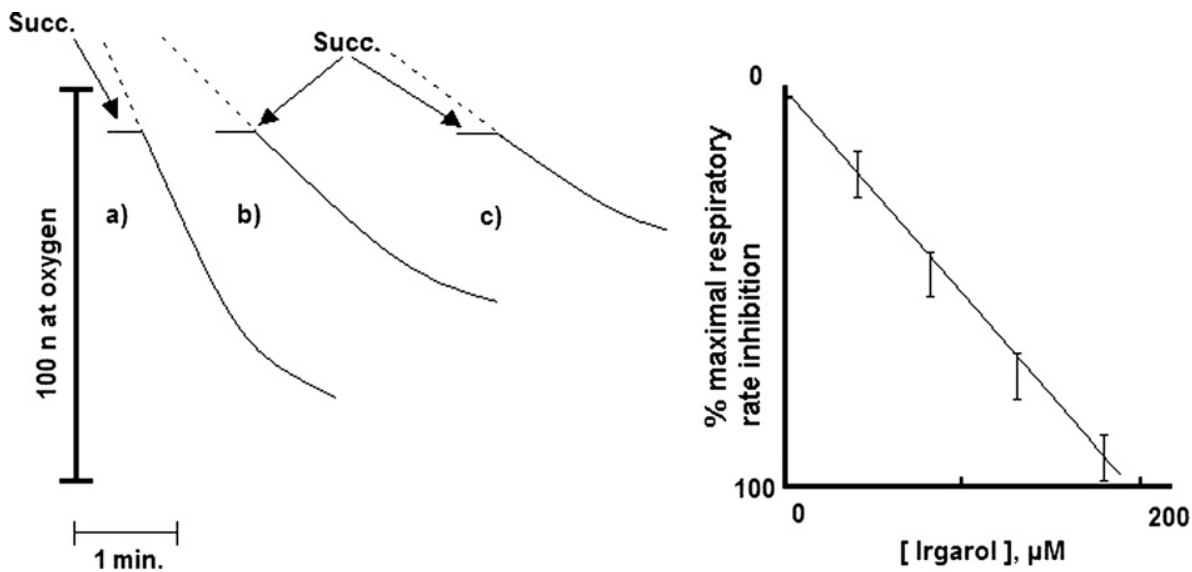

Fig. 2. The inhibition of the respiratory rate of mitochondria by Irgarol. The figure shows a typical experiment in which the basal respiratory rate of the mitochondria was measured in the presence of the FCCP uncoupler and in the presence of varying amounts of Irgarol. In a) no Irgarol was present, but in b) and c), increasing amounts of Irgarol were present. The initial respiratory rate (the rate after the addition the succinate) was reported in the graph. Each experiment was the mean value of four experiments. Medium composition: $0.25 \mathrm{M}$ sucrose, $10 \mathrm{mM}$ Hepes-Mops pH 7.4, $2 \mathrm{mM} \mathrm{MgCl}, 0.5 \mathrm{mM}$ EGTA, $1 \mu \mathrm{M}$ FCCP. The final concentration of mitochondria was $0.5 \mathrm{mg} \mathrm{ml}^{-1}$. The succinate $(2 \mathrm{mM})$, was added 3 min after the addition of the mitochondria and the Irgarol was added immediately after the mitochondria. Similar experiments were performed using Glutamate/Malate ( $2 \mathrm{mM})$ and ascorbic acid (in the presence of ( $0.4 \mathrm{mM}$ TMPD as catalyser) as reducing substrate. In this case no inhibition of the RC was observed.

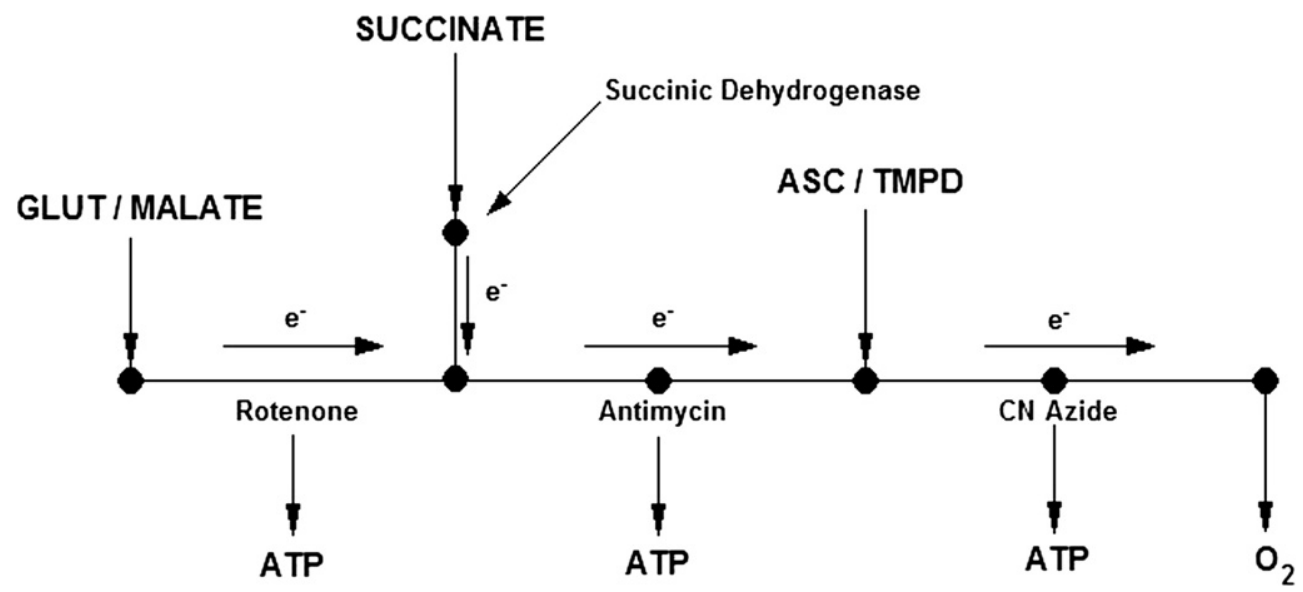

Fig. 3. The respiratory chain in mitochondria. 
This inhibition, however, cannot be responsible for the ATP synthesis inhibition, since in the absence of Cyclosporine it occurs with a lower dose of Irgarol.

\subsection{The ATPase}

This enzyme catalyses the ATP synthesis and, consequently, the inhibition of the ATPase gives rise to an equivalent inhibition of the ATP synthesis.

In order to verify whether the Irgarol inhibits the ATPase, the acidification process which accompanies the ATP hydrolysis (ATP $\rightarrow$ ADP $+\mathrm{P}_{\mathrm{i}}+\mathrm{H}^{+}$) was followed.

Fig. 4 shows the acidification which accompanies the ATP hydrolysis.

The addition of the Irgarol has no consequences regarding the acidification rate.

For the purpose of comparison, the effect of Oligomycin, the classical ATPase inhibitor, is shown (Fig. 4).

We conclude that, since the Irgarol does not induce ATPase inhibition, this step is not responsible for the ATP synthesis inhibition by the Irgarol.

\subsection{Membrane proton permeability}

An enhancement of the membrane proton permeability inhibits ATP synthesis, since the chemiosmotic hypothesis requires impermeability of the membrane to the protons.

The enhancement of the proton permeability could be due either to the presence of uncouplers, which permeate the membrane to the protons, or to a detergent effect, which induces a membrane leak in all ions, the protons included. In addition, an enhancement of the membrane

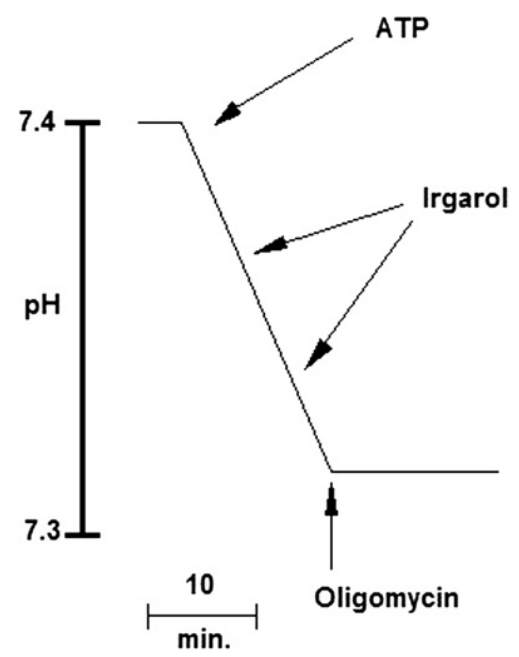

Fig. 4. ATP hydrolysis in mitochondria. The rate of ATP hydrolisis in mitochondria was measured following the $\mathrm{pH}$ change which accompanies the ATP $\rightarrow$ ADP $+\mathrm{P}_{\mathrm{i}}+\mathrm{H}^{+}$reaction. Since the Irgarol had no effect on the ATP hydrolysis and, consequently, on the ATPase, the effect of Oligomycin, a potent ATPase inhibitor, was demonstrated here. Medium composition: $0.25 \mathrm{M}$ sucrose, $0.1 \mathrm{mM}$ Hepes-Mops pH 7.4, $1 \mathrm{mM}$ ATP, $1 \mu \mathrm{M}$ FCCP. Mitochondria $0.5 \mathrm{mg} / \mathrm{ml}$. The concentration of Oligomycin was $1 \gamma$. permeability can be due to the opening of a transmembrane pore which induces the passage of big molecules, the protons included (Bernardi, 1999).

With regard to this last point, the opening of large size membrane pores can be evidenced by means of swelling experiments: the opening of the large size pores induces the entry of sucrose into the mitochondrial matrix from the resuspending medium, but the influx of sucrose is not balanced by an equivalent $\mathrm{K}^{+}$efflux and therefore, is accompanied by the entry of water and a consequent swelling ("colloid osmotic effect" (Bernardi, 1999)).

This swelling can easily be monitored using a spectrophotometer since, at $540 \mathrm{~nm}$, an absorbance quenching occurs (Bernardi, 1999). The Fig. 5a gives an example of this phenomenon which was induced using phenylarsine, a classical inductor of the opening of the pore (Bernardi, 1999). The phenomenon is inhibited by cyclosporine (dotted line). Irgarol (Fig. 5b) does not induce any swelling and we have therefore concluded that the compound does not induce the opening of large size pores.

It should be noted that many compounds bring about the opening of small size pores (Ichas et al., 1997; Ichas and Mazat, 1998). In this situation, the phenomenon, which is cyclosporine-sensitive, is not evidenced by means of swelling experiments, since the pore does not allow for the passage of large molecules such as sucrose, but only small molecules such as the protons. Therefore, the opening of small size pores can be evidenced by other procedures as below discussed.

Two possibilities remain:

An uncoupling effect and a detergent effect.

Uncouplers are chemical compounds which enhance the proton permeability of the membrane. They are weak acids, or bases, and this mechanism has been explained by means of the chemiosmotic hypothesis.

The uncoupling mechanism of 2,4-dinitrophenol (DNP) was first examined. The theory (Mitchell, 1979) proposes that the weak acid DNP enters the mitochondria and tends to accumulate inside, in the electroneutral form, the driving force being the alkaline-inside $\mathrm{pH}$. Once inside, the negative-inside potential extrudes the phenate ion, thus giving rise to a cyclic process in which, at any cycle, a proton is extruded.

In order to assess the presence of uncouplers many procedures can be utilised.

As regards our experiments, since Irgarol does not induce an ATPase inhibition (Fig. 4) we utilised ATP hydrolysis for the monitoring of an uncoupler. The ATP hydrolysis can easily be followed by means of a $\mathrm{pH}$ meter (see above the experiments described in Fig. 4).

The addition of the ATP to the mitochondria induces the formation of a $\Delta \mathrm{pH}$ and of $\Delta \psi$ as the process the ATP synthesis is reversible. The formation of $\Delta \mathrm{pH}$ and of $\Delta \psi$ therefore induces a lowering of the rate of ATP hydrolysis, as the $\Delta \mathrm{pH}$ and $\Delta \psi$ are forces which oppose the ATP hydrolysis. For this reason, in the experiment concerning ATP hydrolysis (Fig. 4), an uncoupler (FCCP) was 

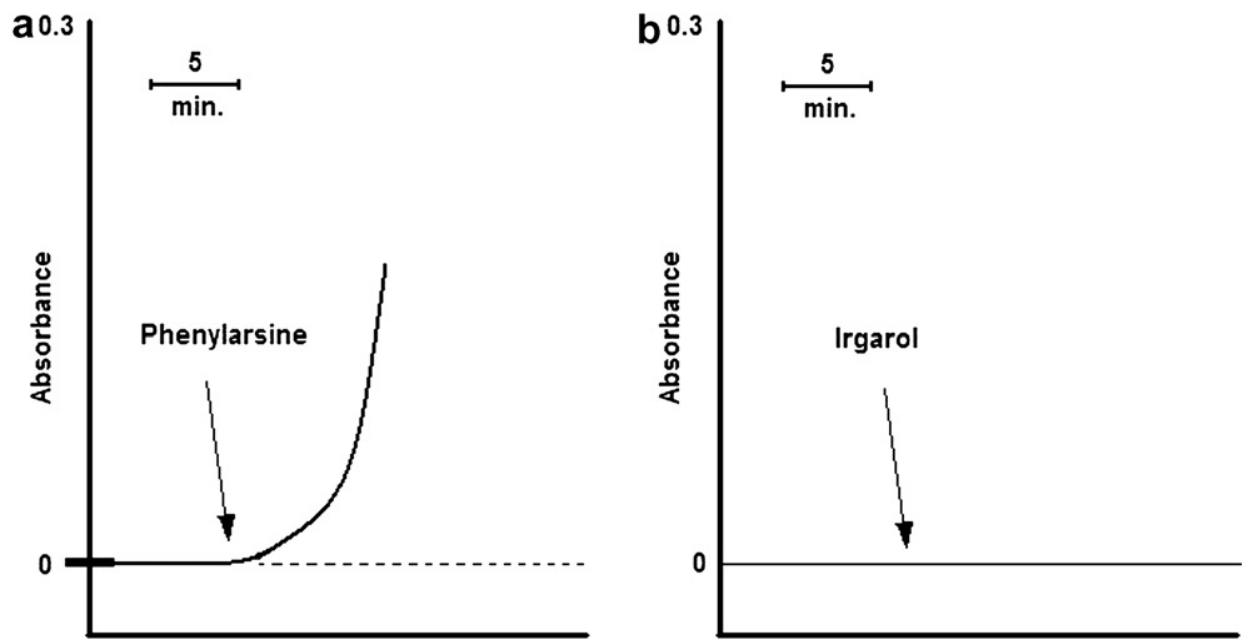

Fig. 5. Irgarol does not induce mitochondrial swelling. The spectrophotometer was adjusted at zero absorbance after the addition of the mitochondria $\left(0.5 \mathrm{mg} \mathrm{ml}^{-1}\right.$, final concentration) to the medium $(2.5 \mathrm{ml})$ Medium composition: $0.25 \mathrm{M}$ sucrose, $10 \mathrm{mM}$ Hepes-Mops pH $7.4,0.5 \mathrm{mM}$ EGTA, $2 \mathrm{mM}$ $\mathrm{MgCl}_{2}, 2 \mathrm{mM}$ succinate. Cyclosporine, when present (dotted line), was $1 \gamma$. In (a), the phenylarsine concentration was $0.01 \mathrm{mM}$. In (b), a typical experiment is shown. In this experiment, the Irgarol was added up to a concentration of $0.2 \mathrm{mM}$.

a

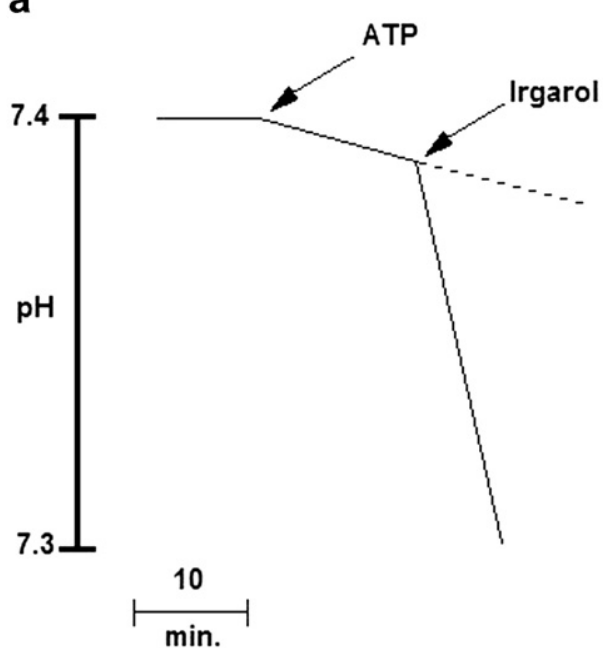

b

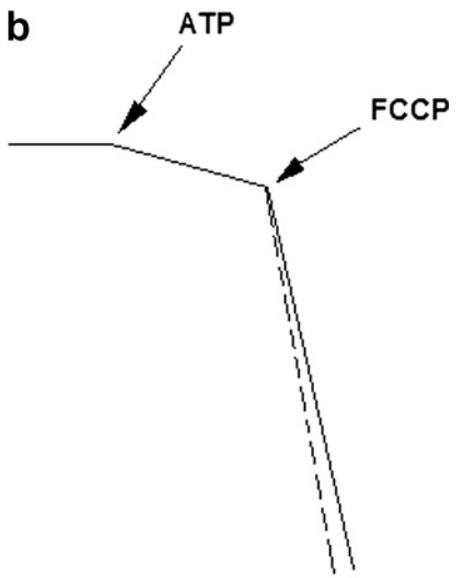

Fig. 6. Irgarol, like FCCP, stimulates the rate of ATP hydrolysis. In experimental conditions which were the same as those in Fig. 4, the rate of ATP hydrolysis was stimulated by the addition of Irgarol (a), and of FCCP (b). However, as regards the Irgarol, the stimulation was inhibited by $1 \gamma$ Cyclosporine (dotted line), while in the case of FCCP (b, dotted line) this does not occur. This supports the hypothesis that the stimulation is due to the opening of a cyclosporine-sensitive pore and not to an uncoupling or to a detergent effect.

added as only the ATPase efficiency was under examination. In fact, an uncoupler collapses the $\Delta \mathrm{pH}$ and $\Delta \psi$, consequently stimulates ATP hydrolysis providing that, as in the case of Irgarol, no inhibition of the ATPase occurs (Fig. 4).

Fig. 6 shows an experiment performed using FCCP (b) which is a potent uncoupler, and with Irgarol (a).

The comparison between the two experiments seems to indicate that Irgarol is a uncoupler. If, however, the experiment is performed in the presence of cyclosporine, there is no evidence of stimulation by Irgarol (dotted line in a). This behaviour indicates that the stimulation is due to neither an uncoupling nor a detergent effect (since an uncoupler and a detergent are not sensitive to the presence of cyclosporine; see Fig. 6b, dotted line), but to the opening of a cyclosporine-sensitive pore induced by Irgarol. Since we have already observed that Irgarol does not induce swelling, one possibility remains, that it induces the opening only of a small size cyclosporine-sensitive pore.

\section{Conclusions}

The experimental evidences show that Irgarol is not an uncoupler, a detergent or an inhibitor of the ATPase. The RC inhibition induced by Irgarol (Fig. 2) cannot be responsible for the ATP synthesis inhibition, since the doses of Irgarol necessary to inhibit the RC are higher than those necessary to inhibit the ATP synthesis (Fig. 1). This 
suggests the presence of another reason which causes ATP synthesis inhibition.

Since the ATP synthesis inhibition is cyclosporine-sensitive (Fig. 1), this inhibition should be due to the opening of a cyclosporine-sensitive pore, but, since the opening of large size pores can be excluded by the swelling experiments, this induces to conclude that the opening of small size pores is responsible (i.e. it requires the lowest dose) to inhibit the ATP synthesis.

\section{References}

Bernardi, P., 1999. Mitochondrial transport of cations: channels, exchangers and permeability transition. Physiol. Rev. 79 (4), 1127 1155.

Bragadin, M., Pozzan, T., Azzone, G.F., 1980. Kinetics of $\mathrm{Ca}^{++}$carrier in rat liver mitochondria. Biochemistry 18, 5972-5978.

Bragadin, M., Pavoni, B., Scutari, G., Manente, S., 2005. An in vitro study of the interaction of Sea-Nine with rat liver mitochondria. Environ. Toxicol. Chem. 24, 1074-1078.
Dahl, B., Blank, H., 1996. Toxic effect of the antifouling agent Irgarol 1051 on periphyton communities in coastal water microcosms. Mar. Poll. Bull. 32, 342-350.

Evans, S.M., Birchenough, A.C., Brancato, M.S., 2000. The TBT ban: out of the fryng pan into the fire? Mar. Pollu. Bull 3, 204-211.

Ichas, F., Joauville, L.S., Mazat, F., 1997. Mitochondria are excitable organelles capable of generating and conveyng electrical and calcium signals. Cell 348, 1145-1153.

Ichas, F., Mazat, J.-P., 1998. From calcium signaling to cell death: two conformations for the mitochondrial permeability transition pore: switching from low- to high-conductance state. Biochim. Biophys. Acta $1366,33-50$.

Lowry, O.H., Rosemberg, N.J., Farr, A.L., Randall, R.J., 1951. Protein measurement with the folin phenol reagent. J. Biol. Chem. 193, 265275.

Mitchell, P., 1979. Keilin's respiratory chain concept and its chemiosmotic consequences. Science 286, 1148-1159.

Okamura, H., Watanabe, T., Aoyama, I., Hasobe, M., 2002. Toxicity evaluation of new antifouling compounds using suspension-cultured fish cells. Chemosphere 46 (7), 945-951.

Panaguola, B., Panayota, M., Iliopoulou-Georgudaki, J., 2002. Acute toxicity of TBT and Irgarol in Artemia salina. Int. J. Toxicol. 21 (3), 231-233. 